\title{
Professional translational research: a new hybrid paradigm in early drug discovery
}

\begin{abstract}
While industry makes cuts to early drug discovery research, the demand for innovation in the pursuit of novel medicines continues to grow. Who should fill this gap? Academia clearly is a rich source of innovation but how can new basic research concepts find their way into industrial application? A new paradigm for early drug discovery involves professional translational research centers, which function as facilitators and translators at the academia-industry interface, harnessing the strengths of both worlds and leveraging the high innovation potential of academia by using the robustness and efficiency of industry. In this article, the authors discuss the set-up and essential requirements for the successful translation of new drug concepts.
\end{abstract}

In 2011, Bernard Munos was cited as saying, 'the next three years will probably see an implosion of the old model of drug discovery' [1]. Today, early drug discovery (eDD, here defined as the discovery phase up to a solid in vivo proof of concept in a relevant animal model; see Figure 1 displaying the full drug discovery and development process with eDD highlighted in green area) clearly is subject to a substantial structural change with a strong trend of industry leaving and academia pushing into this field bringing along a plethora of challenges. Many recent publications discuss threatening challenges in eDD such as patent cliffs, drying pipelines, the decreased productivity in current pharmaceutical drug discovery and development and how industry can fight these. Why bother? If the pharma industry is not interested in $\mathrm{eDD}$, why should somebody else step in? Undoubtedly, the aging society, as well as global warming and the constant growth of the global population, present serious health challenges. There is a worrying increase in patients having diseases, with no or inadequate treatment options, such as degenerative and infectious diseases. If more and more pharmaceutical companies do not continue to pursue $\mathrm{eDD}$ for these health challenges, because the development cycles are too long or too risky, society as a whole should develop a new model in order to cope with these pressing health issues. Who should fill the gap in $\mathrm{eDD}$ resulting from significant resource cutting in industry? Many ideas and potential solutions have been proposed, including new models involving a strong role for academia in eDD. But very rarely is a recipe or even factual experience described about how it may be put into practice, and how it may be technically accomplished, how it may really work in daily business, particularly, considering the fundamental differences of the two worlds, academia and industry. Based on our long-standing working experience at the interface of academia and industry, this article describes lessons learned, a new paradigm for eDD that really works and delivers, and ideas for further improvements in this field.

\section{Structural change in industrial eDD}

What are the causes for the structural change in industrial eDD, why are companies laying off researchers and closing sites? The standard answers to these questions list the stagnating productivity and innovation in the pharmaceutical industry, the increasing costs for drug discovery and development and the rising regulatory hurdles. The primary reason may well be that nearly any industry has its
Peter Nussbaumer $*, 1$ \& Bert Klebl ${ }^{1}$ 'Lead Discovery Center GmbH, Otto-Hahn-Straße 15,

44227 Dortmund, Germany *Author for correspondence: Tel.: +49 231974 27006; Fax: +49 231974 27039; nussbaumer@lead-discovery.de 


\section{Key terms}

Early drug discovery: Drug discovery phase ranging from target identification up to lead nomination of a patentable compound series demonstrating solid in vivo proof of concept in a relevant animal model.

Translational research center (TRC): Drug discovery incubator organization with integrated drug discovery expertise aiming at (de)validation of basic research concepts and advancing them into marketable assets.

cycles of prosperities and dilemmas and we are facing a natural regulation process. During its golden age, pharmaceutical industry was growing and hiring, but at the end could not deliver at the same rate. Industry set out to do basic research, to invest heavily in target identification, early target validation and -omics approaches, such as proteomics and functional genomics. Much money and resources were invested but the outcome was disappointing, multiplied by the typical lemming behavior in the industry: 'what the others do is validated and has to be good'. Based on individual (serendipity) successes in the golden ages of the pharmaceutical industry, scientific management was introduced in the belief of making drug discovery a mature science and making successes projectable for changing the working environment and attitude [2]. Target-based drug discovery and several hyped technologies were overestimated and none of these really delivered on the short-term economic expectations. Apart from elaborated target identification efforts, a major bottleneck in target-based drug discovery is the proper validation of a novel target. Validation by genetic means has its pitfalls and is only achieved if a specific ligand for this target shows activity in an animal model. To reach proof of concept in vivo, intense basic research may be needed, which is hard to defend in an economically oriented structure like a pharmaceutical company. With 25-35 new drugs - only a few of which are breakthroughs - for US $\$ 140$ billion of annual R\&D spending, the return for society is limited and does not match the high costs of the traditional big pharma model [3]. Small companies can do it cheaper, but in general, the biotech industry has not delivered on the expectations either; overall biotech companies have a high attrition rate mainly due to the fact that the duration of the value generation cycles in eDD do not match the short-term expectations of many investors resulting in chronic underfunding (this seems to become more and more geography dependent on the vastly different financing conditions, that is, in 2014 and 2015 biotech fundraising in the USA has been substantially more effective when compared with continental Europe). Especially in eDD, it has to be accepted that certain pharmaceutical routines are needed for generating high-quality assets as true value and that this process takes time. Value generation in eDD cannot be easily accelerated when budget is a rate limiting factor. The necessity to go for fundraising over and over again and the associated time pressure frequently lead to wrong decision making in the critical phases of eDD, to promote immature products and, consequently, to increased attrition. This statement mostly holds true for asset focused biotech companies and not so much for platform technology or service providing companies. Moreover, we see a clear tendency that well-funded biotech companies, not constantly burdened with the issues discussed above, have a lower attrition rate.

Basically, golden ages of the pharmaceutical industry are over, changes are necessary and the community needs a new business model for eDD.

\section{Academia as a rich source of innovation}

Lack of innovation is frequently stated as a major cause of the dry pipeline in the industry [4,5]. Looking at the issue from a global perspective, the authors strongly disagree, since there is a wealth of innovative new hypotheses and findings continuously reported by academia. So, the basic problem is not lack of innovation, it is rather a lack of exploitation and translation of existing hypotheses into industrial applications, including rigorous (de)validation with specific pharmacological tools. Industry is rediscovering academia as a rich source of innovation in eDD, and several initiatives have been announced by industry to foster direct interaction $[1,6,7]$. However, this requires a fundamental cultural change because the two worlds have been drifting apart substantially during the last decades due to different incentives, attitudes and cultures and loss of mutual respect and trust. Money and expectations alone (which will definitely be different in the two worlds) will not solve the problem. Colocalization of academic and industrial scientists may help but also requires, as does any direct collaboration, openmindedness, trust building and mutual acknowledgment, that is, processes that take time and cannot be enforced.

Direct application of academic research in industrial $\mathrm{eDD}$ is further hampered by the following facts: basic research results often lack robustness [8,9]; industry has been getting increasingly risk averse; industry usually operates with dogmas that often do not tolerate out of the box ideas; academia and industry are two worlds with different culture, language, incentives, mindset and career paths; and the tech transfer offices from academic organizations have limited resources and pharmaceutical experience needed to handle the large variety of potential inventions and raise sufficient interest of their demanding industrial counterparts. 


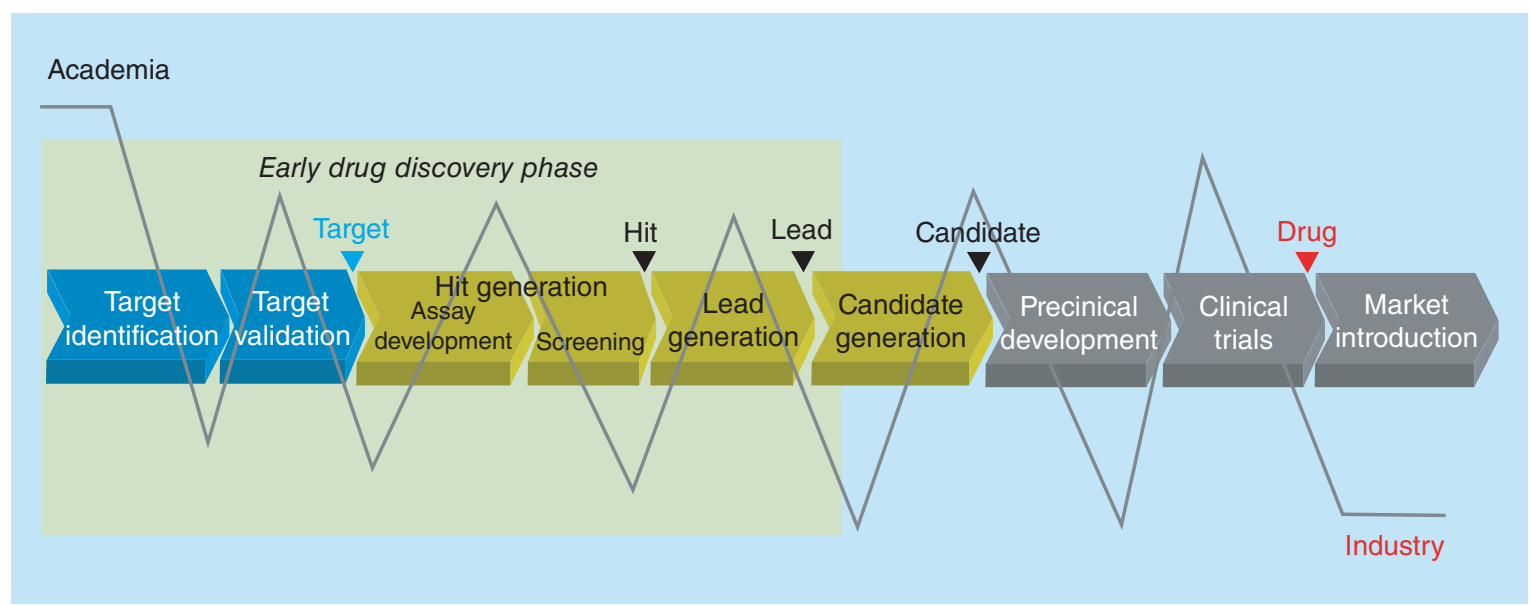

Figure 1. Potential contributions of particular strengths of academia as well as industry to the drug discovery and development process.

\section{Academic drug discovery}

The contributions of academia to drug discovery and marketed therapeutics in general are undisputed. The buzzwords 'academic drug discovery' have been used very broadly for many different efforts and set-ups originating from academia, ranging from a research group operating a screening robot or a chemistry lab up to an association of many academic groups with complementary know-how and skills together representing many essential aspects of eDD. While pushing more and more into eDD, academia usually still lacks the infrastructure and the essential medicinal chemistry know-how for assessing and optimizing in vitro ADMET (absorption, distribution, metabolism, excretion, and toxicity) profiles, that is, physicochemical and biopharmaceutical properties, crucial for eDD [10]. During their work at the interface of academia and industry, the authors have experienced many limitations for successful translation in the pure academic setting, such as the purchase of a screening robot is not enough for high-throughput screening, it also needs skilled, permanent technical staff and a well selected and curated compound collection, adaptation of the assay to automation, robust data generation and analysis, and hardware maintenance; assay development efforts for innovative targets are underestimated and not adequately validated; a chemical probe for target validation must be of the highest quality [11]; integrated drug discovery know-how is important. Cellular studies with insoluble compounds and animal efficacy studies without PK data are worthless; often, we encounter a romantic relationship between an academic investigator and a first nonspecific compound from a primary hit identification effort (also see the pan assay interference compounds dilemma [12,13]); academic institutions are missing project management skills [10] that are important to coordinate and direct the interdisciplinary effort, usually contributed from different labs or even centers, toward the common goal of generating a drug candidate; 'team effort' is differently interpreted in academic and industrial environments; and it is very difficult to deliver industrial quality without prior hands-on experience in an industrial setting.

In need of a new model, academic drug discovery centers (ADDCs) were created and have partially solved these limitations by hiring experienced exindustry staff and/or directors. The remaining issues and questions are: is the academic setup appropriate for $\mathrm{eDD}$ ? Do the current incentive structures in academia work for high-quality drug discovery, which is a must for successful commercialization and later stage partnering to industry? Is academic drug discovery the appropriate solution to substitute for diminishing early research in the pharmaceutical industry? If society is prepared to pay for the learning curve of academics in pharmaceutical research and to receive little or no revenues for a successfully conducted drug discovery program, then the simple answer lies in these purely academic centers, the ADDCs. If the eDD sponsor/investor has any expectation of a return on investment (a financial break-even, long-term sustainability of the eDD organization, or profit), then this is not going to work in an academic environment and it needs a translational research center (TRC)like structure with expertise in industrial-type project execution, business development and professional intellectual property management. Regardless of the (not)-for-profit model, every investor should be prepared to understand that the result of eDD, a proof of concept molecule, is an excellent tool for the validation of the therapeutic hypotheses but still 6-8 years away from a drug in the market. Quite logically, society will benefit from an unbiased effort in funding eDD, as 
long as the funding is driven by medical need instead of potential future commercial income from a novel drug (candidate).

\section{Ideal scenario for eDD \& a fully functional new paradigm: professional translational research}

What would a better model look like? Evidently, both academia and industry can significantly contribute with specialized expertise to drug discovery and development in general (Figure 1, particular strengths represented as peaks in the grey line) and to eDD (area highlighted in green in Figure 1) specifically. For example, within the eDD phase, academia may account for target identification, sophisticated biological assays, tools in assay development, innovative chemistry and specialized animal models; industry's strengths are in robust validation of hypotheses, tool generation, solid assay development, high-quality screening collections, routine compound profiling and animal testing. But, frequently, it is about bits and pieces, modules of specialized expertise that are not at all interconnected. Potential academic contributors, if not a fully integrated part of an ADDC, usually work in independent research groups and entertain some loose cooperation with colleagues from other faculties, while in industry, interdisciplinary teamwork is fully implemented as an admitted success factor. Furthermore, there are two types of innovation: quantum leap and incremental innovation. Some of the recently approved drugs are breakthroughs, but many are still of marginal innovation. Both types of innovation are needed in drug discovery, but, obviously, cannot be successful under the same roof [2]. Quantum leap innovation is killed by the industrial setting and regulations; the chances for serendipity and breakthrough findings are substantially higher in an academic environment [2]. On the other side, industry houses robust processes to reliably deliver solid incremental innovation. In the past, both industry and academia worked within their own worlds and tried to overcome their shortcomings and deficits by investing effort and money in areas that did not really fit their setting and which they could not fully master: drug discovery in academia and basic research in industry. In doing so a lot of money was wasted.

In the vision of the authors, the ideal scenario for $\mathrm{eDD}$ is to bring the strengths of both worlds together, operating like clockwork to leverage the high-innovation potential of academia and the robustness of industrial processes (Figure 2). In such a collaboration mode, everyone can focus on building on and further developing his/her strengths instead of wasting efforts in eliminating weaknesses. In this context, it is important that academia should not be industrialized, but academic researchers should have some understanding of the risks inherent in preclinical drug discovery and of the data quality required for eDD [14]. On the other hand, industry should not compete with academia in pure basic research but rather should support academic research. Mutual trust and respect are strongly demanded! Based on the current status, successful direct collaboration would need a substantial cultural change to overcome the differences of the two worlds in language, attitudes and incentives - a very unrealistic scenario on a short-time scale and broad scope.

Professional TRCs or drug discovery incubators have emerged as a new paradigm for eDD and are

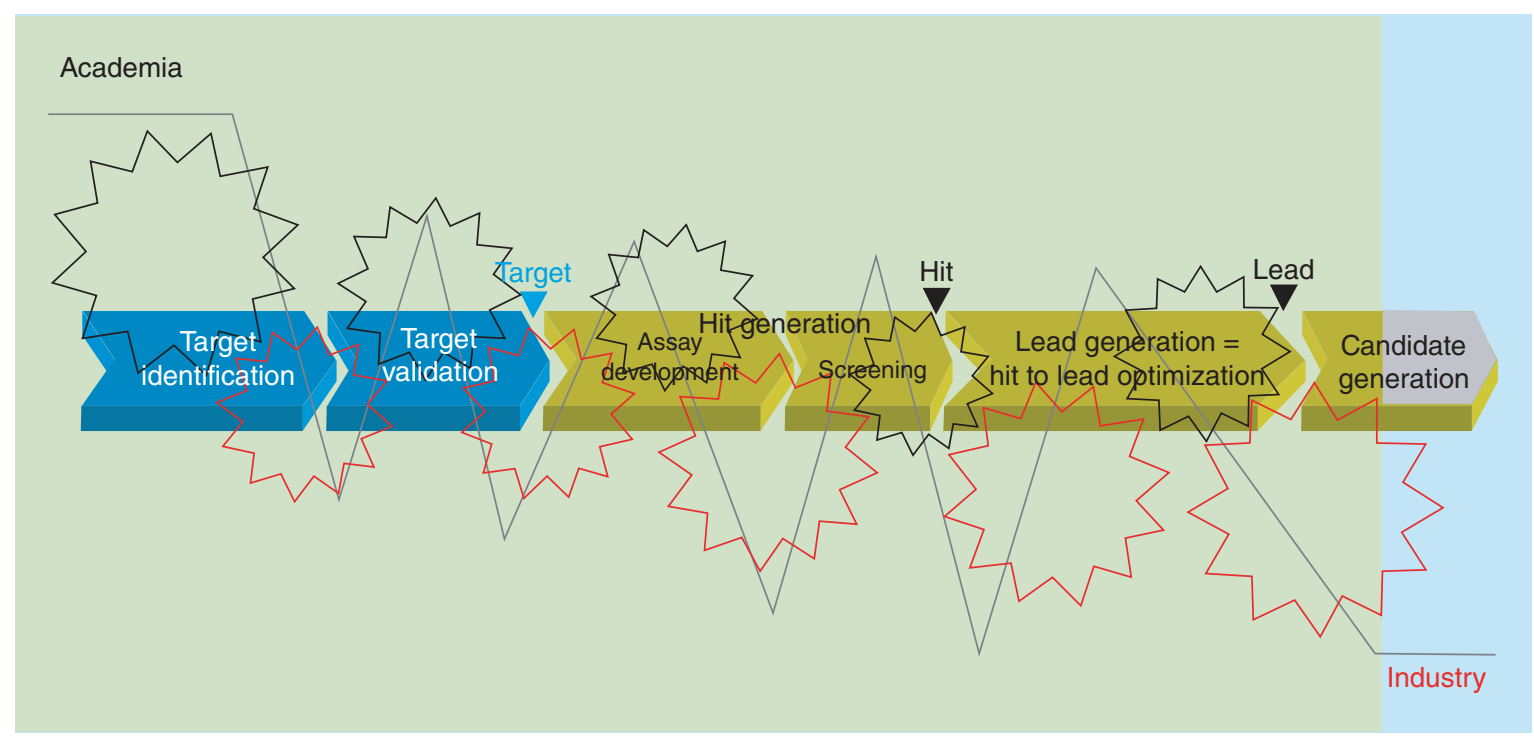

Figure 2. Ideal scenario for early drug discovery; clockwork-like collaboration of strengths of both academia and industry. 
addressing all of these points coming close to the described ideal scenario of eDD. There is some overlap with ADDCs, but also some important enhancements to most of them - and what exactly constitutes an ADDC is loosely defined [15]. The term 'translational research' has also been interpreted equivocally in the past [16], but the authors believe that this is the best descriptive term for what they do and want to propagate. The authors consider the professional TRC to be a 'wet technology transfer incubator' that acts as facilitator, mediator and translator between academia and industry, collaborating with both at eye level and being run by experts in drug discovery. The prime goals of the TRC are to provide a high-quality, developable material basis for new therapies so that patients can benefit from the high-innovation potential of basic research (novel targets and mechanisms); and to derisk innovative hypotheses. Depending on its sponsor and/or investor, the eDD organization will either have a more stringent focus on commercialization or approach novel projects from an academic perspective primarily driven by knowledge generation. As of today, both such concepts have been realized for either commercialization driven models [15], where we see organizations like CD3 of the KU Leuven (Leuven, Belgium), Medical Research Council Technology (London, UK), Cancer Research Technology (London, UK) and the Lead Discovery Center GmbH (Dortmund, Germany) as examples, or de-risking models, such as the Center for Drug Research and Development (Vancouver, Canada). This requires advancing basic research results into potentially marketable assets or, from an application perspective, invalidating basic research findings. If commercialization is the goal, then such early academic assets have to be incubated and translated by professional drug development. Mediating and translating different attitudes, goals, languages and needs among partners are among the important tasks of the TRC. These aspects include advice and consultancy to academic researchers with regard to drug discovery and development processes and requirements such as drug properties, critical project flowchart and robustness of test systems. As an important side effect, TRCs are providing high-quality research tools to academia enabling further basic research and publications on a solid basis.

While every innovative project has its peculiarities, we see the following general requirements to be absolutely crucial for success of a TRC:

- Fully integrated, professional in-house drug discovery expertise;

- Open-mindedness and mediator qualities;
- Close active collaboration with the academic principal investigators (PIs);

- High-quality standards;

- Collaboration with industry instead of competition.

In order to be able to accomplish the tasks and to achieve the objectives, in-house integrated drug discovery involving all relevant disciplines, that is, biochemistry, automation and compound management, biology, medicinal chemistry, pharmacology and project management, is a must. For success and for goaloriented progress, particular to highly innovative eDD projects, it is critical that the design of all key experiments and interpretation of results are done by experienced in-house experts and, whenever appropriate, together with the academic PI. Otherwise, there is a risk of becoming dependent on interpretations of third parties. While the authors believe that it is important that the flowchart-critical assays are best run internally, all wet experiments do not necessarily have to be performed at the TRC or by the academic partner; parts of those can and should be outsourced to keep the organization slim and the efficiency high. The key staff of a TRC has to be open-minded scientists and managers with industry experience. Working at the interface and, thus, also talking and collaborating with industry requires to know what industry expects and needs with respect to products, key results, robustness and quality. On the other hand, open-mindedness is key to not blocking innovative ideas and hypotheses by following dogmas or perceived barriers. Innovation cannot be predicted, but needs a creative environment and time to happen. Based on the experience of the authors, they have identified the tight, active collaboration with the academic originator, that is, the principal investigator, their group and the surrounding (academic) network, as one of the most important success factors for translational research. In a strict project handover, much know-how including many existing results are lost since they look less important at first glance. When the PIs stay involved they do not have to give away their babies and they remain coresponsible and motivated. This constitutes a clear win-win situation on the scientific and the commercial side as some project costs can be saved due to contributions of the PI, and he/she receives a fair share in exchange upon commercialization.

A TRC certainly benefits from being affiliated with an academic organization (vide infra, paragraph on sustainability), but it has to be able to operate separately in order not to get trapped in the academic incentive and salary system and to guarantee independent decision making. Similarly, the TRCs should not be 
governed by industry. Quality data should drive eDD, not publication record, strategy or controlling. Careful project management helps to base decisions on highquality results, to progress projects with a reasonable goal-orientation, to adjust goals based on new insights and to ensure efficient use of funding. With commercialization and further development as main goals, it is clear that the TRC has to deliver high-quality data and compounds including robustness, reproducibility and consistency of results. Low reproducibility of published data has been raised as a strong concern $[8,9]$. The authors do not want to engage in a discussion of the causes, but they have observed that this is not true for most of the projects that they have taken up into their portfolio with wet experiments. The previously mentioned continuous, close interaction with and coresponsibility of the PIs ensure a much higher reproducibility rate of seminal results as an important basis of the respective projects. Arguable and singular results have to be jointly discussed, challenged and (in) validated in a robust manner in order to justify further efforts and investments. But, again, this has to happen without a strong bias, and our approach is very pragmatic; we prefer to design and perform a decisive experiment instead of discussing at length whether it is in line with existing doctrine or hypothesis. This approach leads to continuous risk reduction or pausing of the project. The integrated set-up, the proximity to academia and the size of a TRC fosters creativity, flexibility and motivation to overcome scientific and technical hurdles with unprecedented solutions, thus forming an ideal basis for exploring innovative therapeutic concepts. Functional TRCs aim at collaborating and not competing with industry (pharma as well as biotech). This is guaranteed by working on innovative, novel science and seeking advice from and interaction with the industry. The industry has substantially opened up during the last years [7] (see the efforts around 'open innovation'), and TRCs can take advantage of this by integrating industry modules in the project work. Established examples are collaborations where industry shares corporate compound collections and tools for screening, assay development and validation, respectively, and tests prototype modulators in specialized systems or open innovation platforms. For these types of collaborations, several contractual models are possible ranging from in-kind contributions to option agreements.

\section{Intellectual property, patenting, ownership \& commercialization value}

These topics are very differently interpreted and handled in academia and industry, thus representing one of the biggest areas of misunderstanding between the two worlds. The old myth about academic scientists fearing that industry steals their gold nuggets partly is still alive. Despite recent change for the better, there is still some mistrust and, usually, the self-perceived value and the assessment by industry differ widely. Industry refers to the insufficient level of maturity and demands more validation experiments. This causes frustration at the academic side, knowing how much time and dedication already was spent on the project and that they cannot move their work into potential application by themselves. There is no real market place for basic research results and this is exactly the stage where the TRCs step in and help PIs to validate and translate their findings and hypotheses into assets with commercial value. Moreover, TRCs are instrumental in developing appropriate filing strategies for the incubated projects. The motivation and strategy for filing patent applications in academia is driven by the peer pressure to publish the best results and a perceived potential value in these findings. Priority applications are filed just before the scientific papers on the same matter are submitted; frequently, in the priority year no followup experiments are performed to substantiate the filing and to exemplify the scope, a routine process in industry. Thus, from a commercialization point of view, the filing was too early due to insufficient coverage of the claims (particularly true for filings on chemical matter with biological activity). Based on initial interest of industry and hope, applications are processed further until the fees get high and the early priority date makes them decreasingly attractive for a potential commercial partner, finally resulting in abandonment. Collaboration with a TRC can avoid frustration and waste of money. One may argue that the involvement of a third party (TRC) further complicates the issues regarding intellectual property, ownership and value. However, the TRC in its mediator role provides professional advice, coordination of the patent strategy, and design as well as performance of experiments to substantiate the invention. This substantially increases the value of the assets and the chance of application and commercialization for the direct benefit of the involved PI; the active continuous involvement of the PIs in the projects incubated at TRCs assures their co-ownership and interests. Usually, commercialization and publication needs are a bad match, but this problem can be solved by intentionally following two tracks in parallel, one for commercialization and one for publishing [15]; so far this has worked out very well in all our projects. Moreover, we have experienced that the PIs balance the enhanced chance of commercialization of their projects with their publication plans; they also appreciate that results and probes generated at TRCs can lead to additional high-impact papers. Finally, high-quality 
publications can be instrumental in identifying collaboration partners and even interested licensees.

\section{Deliverables \& sustainability}

In a recent review, the economic and organizational sustainability of ADDCs was analyzed [15]. The authors agree with all the tensions and risks revealed by the analysis; their experience and approach to solving the organizational problems, for example, the institutional incompatibility with the academic set-up, have been already described above. In this section, the authors discuss deliverables, measures of success and economic sustainability. To manage the expectations of potential eDD investors, they first want to share some thoughts about deliverables of TRCs. Emphasizing that a TRC needs a professional set-up and industry experienced people calls for business-oriented success criteria. Indeed, commercialization events are critical deliverables, and together with licensing revenues and patent filings they can be easily measured. But considering the hybrid function of a TRC, there are many more important deliverables such as reliable tool compounds and probes, publications, consultancy and training that enable them to perform better basic science. Scientific success is measured by robust and unequivocal validation or invalidation of hypotheses in appropriate models, preferably in relevant animal models. While the scientific merit of solid invalidation is certainly high, it is impossible to generate a return on this, and even a positive proof of concept in animals does not automatically lead to economic success. The same is true for other soft criteria and deliverables such as high customer satisfaction in both directions, the academic PI and the licensee, but may have a significant impact on follow-up collaborations and business.

Since there will be a continuous need for high-quality eDD in the future, it is the belief of the authors that a TRC should be based and advanced on long-term commitment and sustainability. The very long development cycles and high probability of failure in drug development make this very challenging and require a long phase of initial funding and a lot of stamina. Moreover, industry does not yet fully appreciate the commercial value of robust de-risking of innovative therapeutic concepts. Yet, it is very clear to the authors that the entire drug discovery market is segmented along the pharmaceutical value chain. Pharmaceutical industry and (venture) capital investors primarily look for the biggest jumps in value creation, which certainly take place after launching a novel therapy onto the market. Looking at the phases before product launch, significant proof of concept in a clinical Phase II trial combined with improved efficacy over the gold standard therapy will immediately lead to commercialization. eDD is still years away from clinical trials; nevertheless, proof of concept for a novel (with regard to intellectual property) lead series in a relevant therapeutic animal model is the first important inflection point for value generation, although being still approximately 3 years away from a Phase I clinical trial. If based on appropriate experiments and solid data, the further optimization of such lead series is of limited risk and, therefore, such a project can be commercialized at significant, albeit reduced compared with a clinical Phase II product, licensing terms. Earlier stage projects, that is, a novel target and mechanism as well as hit matter on such a target/mechanism, cannot be commercialized easily and do not yield favorable licensing terms. Based on these considerations, we see a high-value market for clinical molecules, a mediumvalue market for proof of concept leads, and a lowvalue market for targets/mechanisms and hit matter, respectively. The authors conclude that, for eDD, the medium-value market can be commercially rewarding mid- to long-term and can easily generate enough return to sustain a TRC over a couple of years.

Sustainability can be seen as continuously secured project funding through grants from foundations and public sources or, alternatively, being based on commercial revenues [15] (see section above on the medium- and high-value market). While some ADDCs apparently have achieved sustainability via grant funding, the situation is rather different between the USA and Europe [15]. For-profit TRCs may encounter a considerable economical risk by fully relying on grant funding. The authors strongly believe that professional TRCs will become self-sustaining incubators, based on commercial revenues, provided they get a share of the commercialization revenues; they have a constantly rich source of project proposals; and they have enough initial and continuous funding to work on a broad portfolio until the breakeven is reached. Finally, the TRC should be able to finance its own operating costs. According to the authors' model, the commercialization revenues are shared among the academic contributor (the PI and her/his host university, respectively), the funding institution and the TRC. Amazingly, some public funding organizations are not prepared or even not allowed to take commercialization revenues. The authors think the right way to move forward would be to feed appropriate shares in revenues back to the funding bodies for further revolving investment in drug discovery grants. By the way, the concept of having evergreen funds operating for eDD exists, for example, the Boehringer Ingelheim Venture Fund. This would be a relatively fast return to government and society, increasing the amount of grant money in 
the field. The revenue participation of the academic contributors honors their invention and innovation and motivates them to contribute to and collaborate on new projects. The share for the TRC is justified by the processing and translation of hypotheses into commercializable assets paving the way for sustainability. The authors think that the optimal way in eDD is to pragmatically and unbiasedly work on a broad portfolio of innovative projects and bring each of them to a solid go/no-go decision based on individually adapted criteria. Only a broad portfolio guarantees a certain success rate. A critical budget per project without competition among each other is the next key factor for success. At the stage the authors usually engage in new projects, nobody can judge which projects are the best out of a basket of technically prefiltered cherries. The most promising one may fail with the next experiment; underestimated hypotheses may become breakthrough therapies.

\section{The funding issue}

All articles on academic drug discovery and translational research feature lack of funding as the main issue in the field. eDD is not really within the interest of the venture-capital market, except for the cofinancing of some spin-offs led by academic PIs. The development cycles, but also the prime attitudes of venture capitalists and big pharma in the value maximization model, do not match the needs in the eDD field. As mentioned above, the authors are convinced about the broad portfolio approach, while venture capitalists and pharma want to pick the best cherry out of a basket of sweet cherries - which is not reasonably possible in the phases before animal proof of concept. Basic research is fully funded by governmental and public money and as the logical next step into application the authors see financing of eDD projects through both public and private sources. If industry (co)funds eDD at a TRC, it should not have full control or final say; TRCs should not be the extended bench of industry - this is a main differentiator from a classical service provider for medicinal chemistry. Becoming the lab bench of a pharma company would not leverage the advantages of the TRC setup. Moreover, cost saving and risk aversion of industry have boosted the establishment of TRCs as new paradigm in eDD, provided that society continuously sees the need to support eDD. In the opinion of the authors, there is no lack of money for financing eDD at TRCs; the money is available but either saved to increase short-term profit (industry) or inefficiently used (public). Public funding strategies for translation need to be revised and adapted, particularly in Europe where large and rigid academic consortiums have been funded to perform translational work. A lot of money has been wasted because academic PIs and academic consortia, even when trying hard to reach the translational objective, frequently lack the appropriate $\mathrm{eDD}$ expertise, and the grant money is often abused by the consortium partners to crossfund their internal research, instead of conducting the more 'boring' eDD work. With the establishment of professional TRCs, the authors would suggest a clear restructuring toward significant funding of small consortia in collaboration with or directly involving professional TRCs where the drug discovery and project management expertise is available. Thus, the authors expect a more efficient use of the grant money with the potential to generate revenue streams into academia. There is room as well for additional professional incubators and for public-private partnership models in eDD; this is particularly true in Europe, despite or particularly because of the Innovative Medicine Initiative, since the Innovative Medicine Initiative programs operate to a certain extent against self-sustainability of nonindustry partners. 'All these models put academic researchers at the heart of drug discovery. But they will fail unless more money flows ... into academic labs' [1]. The authors agree with the first statement but translation should run professionally and the money dedicated to eDD should not flow into academic labs. Academic labs will get substantial return for collaborating with TRCs, in the form of validated research tools and shares in commercialization revenues, both of which they can use freely for what they do best, that is, basic research.

\section{Summary \& potential improvements}

eDD expertise has been centered around industry in the past decades. Academic drug discovery has emerged to further advance basic research toward application and to substitute diminishing eDD in industry, but many academic centers are still missing essential elements of eDD. Professional TRCs with experienced experts in key positions provide further enhancements to bring together and synergistically leverage existing, recognized strengths of both academia and industry. One of the functions of a TRC is a mediator and translator role between the two worlds (academia and industry) that requires an extremely balanced and open-minded attitude. It is important to disengage from any artificial, limiting barriers, dogmas and purely strategic considerations. High-quality scientific results shall drive projects fully aligned with appropriate project management guidance and objectives. Equally important are appreciation of each other and mutual trust. It is all about balancing and to apply neither the indus- 
trial nor the academic style alone. This has to be reflected in hiring as well, since the TRC needs personnel who understand and appreciate both worlds. Potential tensions regarding publication versus intellectual property for commercialization are not considered a real issue. The authors have installed both commercial and publication tracks [15].

Though TRCs are coming close to the ideal scenario of eDD, there is still room for improvement, especially for the involvement of industry. In-kind contributions are important elements and can be extended, for example, we see a benefit in industry opening up its animal testing facilities for TRC compound testing. Usually, the licensing interest from industry is triggered by positive in vivo data providing proof of concept of the hypothesis in an animal model, the first inflection point in drug discovery, even when industry has been aware of the hypothesis for some time. The first step before commercialization is retesting of the prototype compound internally under material transfer agreement, frequently in the same animal model. A lot of money could be saved when industry agrees to already do the first in vivo testing and, moreover, the overall data quality may get better. In addition to in-kind, industry shall not have full control over eDD, since the basic research aspects within $\mathrm{EDD}$ are not in line with the decision-making processes in a purely economically driven environment. Companies will benefit either from potential in-licensing candidates or from learning about failures and dead ends encountered in projects and the critical know-how around the project [17]. Reliable invalidation of a hypothesis is as important as validation; however, only positive results can be commercialized. In recognition of professional invalidation, a grant money and award system should be in place. Consequently, the two major products of TRCs are licensing packages for industry (pharma and biotech) and invalidated targets and mechanisms that are disseminated to the community.

In general, rearranging of funding schemes and creating new dedicated funding pots for professional translation [17] enable self-sustaining incubators to generate multiple value for society; more and better new therapies based on highly funded basic research, lower costs for eDD (compared with industry) and development due to lower attrition, and strengthening of basic research through revenues can be achieved.

\section{Conclusion \& future perspective}

The authors do not expect pharmaceutical industry to completely exit eDD, though they expect some more consolidation among mid-size and large compa- nies resulting in further job cuts. Pharma companies will conserve their knowledge in eDD in order to be able to scout for, evaluate and validate licensing and collaboration opportunities. By and large they will keep their activities focused on later stage drug discovery and development. The authors expect a substantial increase of eDD programs in/from academia and at biotech companies in well-funded geographical regions. Further opening and externalization of industry and more interactions with academia will help to build some mutual trust and to narrow the gap between academia and industry, but will not sufficiently solve the translation gap, that is, a routine process to bring basic research results into pharmaceutical development. Academic drug discovery in general will experience a short-term boom as the 'push' from academia is very high, but the authors expect a natural regulation based on real successes versus constraints regarding costs and quality. TRCs with their fully professional but open-minded attitude and mediator function will play an important role in future eDD. Based on the criteria discussed in this article and by collaborating with the pharmaceutical industry, the authors are convinced that TRCs can become selfsustaining incubators serving patients, society and academia. Besides the potential emergence of additional eDD models, well-funded biotech companies will represent key drivers of future drug discovery and development. One of their main roles will be a bridging function between eDD - either based on internal innovation or moving forward assets from TRCs and academic eDD - and industrial later stage development; their set-up allows fast maturation of eDD assets into innovative clinical candidates, raising the acquisition appetite of the bigger pharma companies. Later stage development and market introduction will reside in the main role of big pharma.

\section{Financial \& competing interests disclosure}

The authors have no relevant affiliations or financial involvement with any organization or entity with a financial interest in or financial conflict with the subject matter or materials discussed in the manuscript. This includes employment, consultancies, honoraria, stock ownership or options, expert testimony, grants or patents received or pending, or royalties.

No writing assistance was utilized in the production of this manuscript.

\section{Open access}

This work is licensed under the Attribution-NonCommercial-NoDerivatives 4.0 Unported License. To view a copy of this license, visit http://creativecommons.org/licenses/bync-nd/4.0/ 
Executive summary

Structural change in industrial early drug discovery \& academia as a rich source of innovation

- Early drug discovery (eDD) is subject to a substantial structural change with a strong trend of industry leaving and academia pushing into this field.

- New models for eDD are required to respond to the change.

- Academia is a rich source of innovation as basis for new potential therapies.

- Direct routine application of academic research results in industrial eDD is hampered by several facts that cannot be changed in short term.

- Successful collaboration between academia and industry in eDD needs a cultural change to overcome the differences in incentives, attitude, mindset and culture.

Academic drug discovery

- Academic drug discovery centers (ADDCs) have emerged in consequence of the lack in efficient translation and the push from academia into eDD.

- The term ADDC is very loosely defined and most ADDCs are still missing essential elements or skills required in eDD.

- Moreover, the authors question whether the academic set-up and incentive structures can be appropriated for professional eDD.

Ideal scenario for early drug discovery \& professional translational research centers as a fully functional new paradigm

- Both breakthrough and incremental innovation are needed in eDD, but require different environments and attitudes.

- The authors' vision of the ideal scenario for eDD is combining the recognized strengths of both academia (high innovation potential) and industry (robustness of processes).

- Translational Research Centers (TRC) as professional incubators with experienced experts in key positions represent a new paradigm in eDD.

- TRCs function as mediator and motor in a trilateral collaboration being close to the ideal scenario.

- The following requirements are crucial for success of a TRC: fully integrated professional in-house drug discovery expertise, open-mindedness and mediator qualities, close active collaboration with the academic principal investigator, high-quality standards and collaboration with industry instead of competition.

- Quality data should drive eDD, not publication record, strategy or controlling.

Translational research centers: value generation, deliverables, funding, sustainability \& future perspective

- TRCs substantially increase the value of the assets and the chance of application and commercialization for the direct benefit of the involved PI.

- TRCs are instrumental in developing appropriate filing and publishing strategies meeting both commercialization and publication needs.

- The main goals of TRCs are unequivocal validation/invalidation of scientific hypotheses and commercialization of assets generated together with the academic principal investigator.

- In addition, there are many more important deliverables such as provision of specific tool compounds and probes, publications, consultancy and training.

- Since there will be a continuous need for high-quality eDD, TRCs should be based and advanced on long-term commitment and sustainability.

- Based on shares in the revenues of the commercialized projects, TRCs can become self-sustaining incubators.

- Public funding strategies for translation need to be revised and adapted, particularly in Europe.

- TRCs will play an important role in future eDD generating multiple value for patients, society and academia.

- More intensive involvement of industry with actual work packages will further enhance this eDD paradigm.

\section{References}

1 Cressey D. Traditional drug-discovery model ripe for reform. Nature 471, 17-18 (2011).

2 Schmid EF, Smith DA. Should scientific innovation be managed? Drug Discov. Today 7, 941-945 (2002).

3 Milet $\mathrm{S}$. The next wave of pharmaceutical innovation: an interview with Bernard Munos.

www.forbes.com/sites/medidata

4 Paul SM, Mytelka DS, Dunwiddie CT et al. Hot to improve R\&D productivity: the pharmaceutical industry's grand challenge. Nat. Rev. Drug Discov. 9, 203-214 (2010).
5 Pammolli F, Magazzini L, Riccaboni M. The productivity crisis in pharmaceutical R\&D. Nat. Rev. Drug Discov. 10, 428-438 (2011).

6 Ledford H. Drug buddies. Nature 474, 433-434 (2011).

7 Simpson PB, Reichmann M. Opening the lead generation toolbox. Nat. Rev. Drug Discov. 13, 3-4 (2014).

8 Prinz F, Schlange T, Asadullah K. Believe it or not: how much can we rely on published data on potential drug targets? Nat. Rev. Drug Discov. 10, 712-713 (2011).

9 Dolgin E. Drug discoverers chart path to tackling data irreproducibility. Nat. Rev. Drug Discov. 13, 875-876 (2014). 
10 Nussbaumer P. Medicinal chemists of the 21st century - who are we and where to go? ChemMedChem 10(7), 1133-1139 (2015).

11 Frye SV. The art of the chemical probe. Nat. Chem. Bio. 6, 159-161 (2010).

12 Baell JB, Holloway GA. New substructure filters for removal of pan assay interference compounds (PAINS) from screening libraries and for their exclusion in bioassays. J. Med. Chem. 53, 2719-2740 (2010).

13 Baell JB, Walters MA. Chemical con artists foil drug discovery. Nature 513, 481-483 (2014).
14 Dahlin JL, Inglese J, Walters MA. Mitigating risk in academic preclinical drug discovery. Nat. Rev. Drug Discov. 14, 279-294 (2015).

15 Kirkegaard HK, Valentin F. Academic drug discovery centers: the economic and organisational sustainability of an emerging model. Drug Discov. Today 19(11), 1699-1710 (2014).

16 Fishburn CS. Translational research: the changing landscape of drug discovery. Drug Discov. Today 18(9/10), 487-494 (2013).

17 Ladbury JE, Hall A, Skidmore J. The toolbox is open, but who should pay for the job? Nat. Rev. Drug Discov. 13, 479-480 (2014). 